\title{
Zinc nanoparticles in solution by laser ablation technique
}

\author{
S C SINGH and R GOPAL* \\ Department of Physics, University of Allahabad, Allahabad 211 002, India
}

MS received 26 December 2006; revised 12 April 2007

\begin{abstract}
Colloidal zinc metallic nanoparticles are synthesized using pulsed laser ablation of metal plate in an aqueous solution of suitable surfactant to prevent aggregation. UV-visible absorption, TEM, small angle $\mathrm{X}$-ray diffraction and wide-angle $\mathrm{X}$-ray diffraction are used for the characterization of colloidal zinc metallic nanoparticles. Colloidal nanoparticles are found highly stable for a long time.
\end{abstract}

Keywords. Zinc nanoparticles; colloidal nanoparticles; nano-powder; PLAAM.

\section{Introduction}

Recently, chemists, physicists and materials scientists have shown great interest in the development of new methods for the synthesis of nanomaterials. These materials are important for further miniaturization of electronic devices, nanofluids (Eastman et al 2001) to enhance efficiency of heat engines, as chemical catalysts (Kuang et al 2000; Maye et al 2000) and lasing materials (Huang et al $2001)$ etc. Physical and chemical properties of these materials are highly size dependent. Therefore, it is important to develop novel techniques for the synthesis of smaller and monodispersed nanomaterials. Conventional chemical reduction method uses hazardous chemicals and has no means of controlling size and shape of nanoparticles. Zinc and copper nanoparticles are highly oxidative as compared to silver and gold, therefore, synthesis of these particles in the aqueous media is preferred. Recently, pulsed laser ablation in aqueous media (PLAAM) has provided a new technique for synthesis of size controlled metallic nanoparticles. Metallic nanoparticles in colloidal and powder phase were synthesized using different techniques by several investigators (Mulvaney et al 1993; Macdonald and Smith 1996; Li et al 2004; Darugar et al 2006). Lasers have provided a powerful tool for the synthesis of nanomaterials in both solutions and gas matrices (Hodak et al 2000; Ma et al 2005). Resizing and reshaping of colloidal nanoparticles synthesized by other techniques are also possible through melting and fragmentation mechanism (Geohegan et al 1998; Fujiwara et al 1999; Link and El-Sayed 1999). Synthesis of colloidal silver nanoparticles using laser ablation of metal salt in aqueous media is also reported (Abid et al 2001). Gold (Sibbald et al 1996), silver (Mafune et al 2000), metallic and Ag-Au bimetallic (Lee et al 2001) nanoparticles are produced by laser ablation of

*Author for correspondence (spectra2@rediffmail.com) gold, silver and its alloy plates, respectively in an aqueous media of suitable surfactant. Synthesis (Rakshit and Budhani 2006) and magnetic characterization of $\mathrm{Co}-\mathrm{Pt}$ nanoparticles are reported by laser ablation of Co-Pt bulk alloy in aqueous media. The present study deals with colloidal zinc nanoparticles, synthesized by pulsed laser ablation in aqueous media (PLAAM) technique, and its characterization. Production of $\mathrm{ZnO}$ nanoparticles is also reported in small amount. Synthesis of colloidal $\mathrm{Zn} / \mathrm{ZnO}$ nanoparticles using laser ablation technique is not reported elsewhere, to the best of our knowledge. Recently, synthesis and characterization of $\mathrm{ZnO}$ nanoparticles in aqueous media using laser ablation technique has been reported by Singh and Gopal (2006).

\section{Experimental}

Zinc nanoparticles were produced by pulsed laser ablation of a piece of zinc metal in an aqueous solution of SDS (Glaxo Smith Kline) using double distilled water as a solvent. The slice of zinc rod $(99.99 \%$, Spec-pure Johnson Mathey) was placed at the bottom of glass vessel containing $10 \mathrm{ml} 0.05 \mathrm{mM}$ aqueous solution of SDS. A piece of metal was irradiated by the focused output of the second harmonics of pulsed nanosecond $\mathrm{Nd}$ : YAG laser (Spectra Physics Inc., USA) operating at $10 \mathrm{~Hz}$ frequency, $100 \mathrm{~mJ}$ energy, with a lens having a focal length of $250 \mathrm{~mm}$ for $60 \mathrm{~min}$. Experimental arrangement is shown in figure 1. Plasmon absorption spectrum was recorded using UVvisible Sistronics double beam spectrophotometer 2203 Smart. Larger particles and free SDS appearing as residue were separated from colloidal solution by $5000 \mathrm{~g}$ centrifugation. Vacuum evaporation of colloidal solution at $60^{\circ} \mathrm{C}$ was used to raise the concentration of particles in the solution. Size of zinc nanoparticles was determined by small angle X-ray diffraction (SAXD) while synthesis of zinc phase was confirmed with wide angle X-ray di- 
ffraction pattern using Rigaku D/Max 2200 diffractometer with $\mathrm{CuK} \alpha$ radiation at $\lambda=1.5406 \AA$. SAXD was recorded between $0^{\circ}$ and $5 \cdot 0^{\circ}$, while wide angle X-ray diffraction pattern was obtained in the range of $0-70^{\circ}$. Guinier plots were used to measure the diameter and distribution of nanoparticles from SAXD pattern. For TEM measurement, one drop of colloidal solution of zinc nanoparticles was placed at the carbon coated copper grid and dried in vacuum oven at $320^{\circ} \mathrm{K}$ temperature. The prepared grid was washed with water to remove free SDS particles and placed below the objective of E.M-C.M-12 (Philips) tunneling electron microscope operating at $200 \mathrm{keV}$ to record TEM image of the same.

\section{Results and discussion}

Figure 2 shows UV-visible plasmon absorption spectrum of zinc nanoparticles produced by laser ablation in aqueous

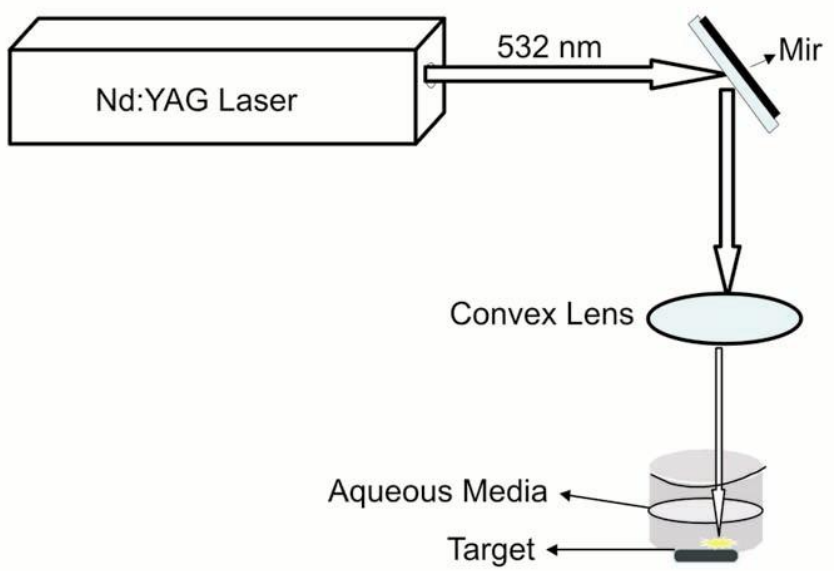

Figure 1. Experimental arrangement.

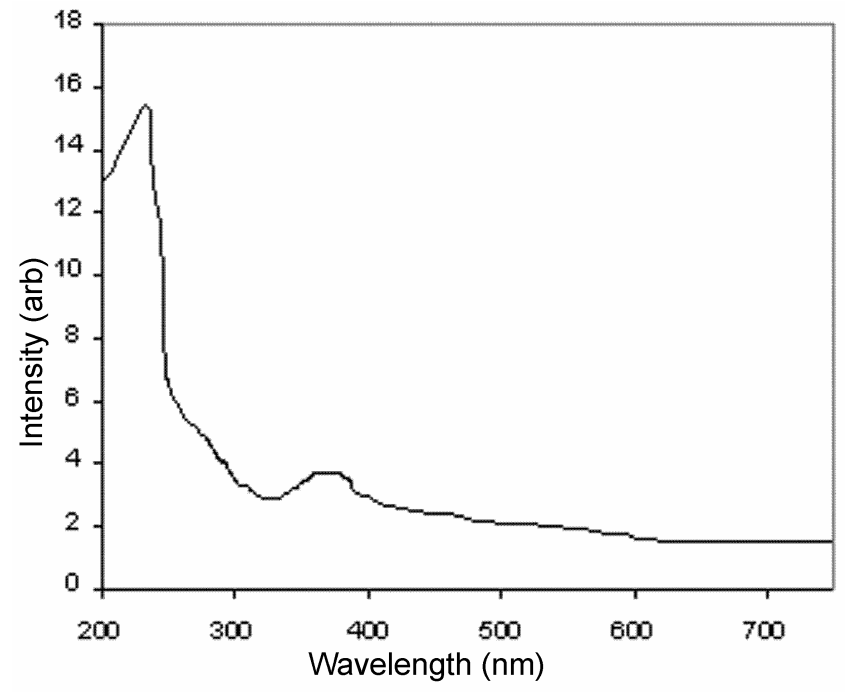

Figure 2. Absorption spectrum of zinc nanoparticles. media and TEM image of colloidal zinc is presented in figure 3 . The absorption spectrum has a sharp peak centred at $232.4 \mathrm{~nm}$ with a long tail towards longer wavelength side, which is similar to the absorption band of cadmium, of the same group, nanoparticles experimentally reported by Henglein and theoretically supported by Mulvaney (Henglein et al 1992; Mulvaney et al 1993). Synthesis of zinc nanoparticles is also verified using small angle X-ray diffraction (SAXD) and TEM experiments, which results in almost the same value of $14.7 \mathrm{~nm}$ for all the average sized particles. Wide angle X-ray diffraction pattern contains an intense peak at $2 \theta=20 \cdot 4^{\circ}$, which is the characteristic of zinc phase. X-ray diffraction pattern is shown in figure 4, which includes SAXD pattern in the inset. Absorption spectrum also contains a small hump centred

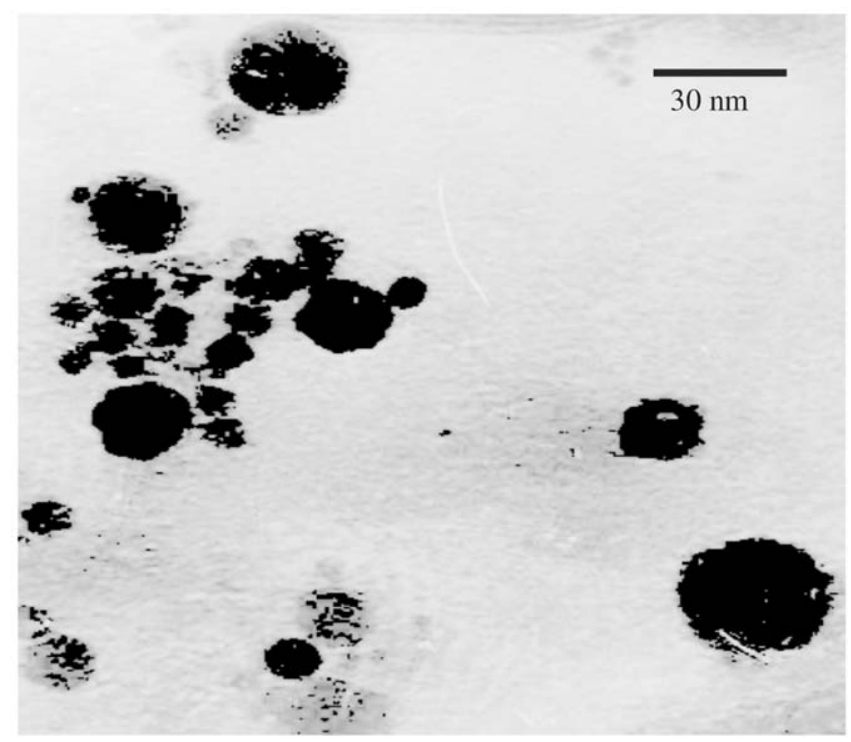

Figure 3. TEM images of colloidal zinc nanoparticles.

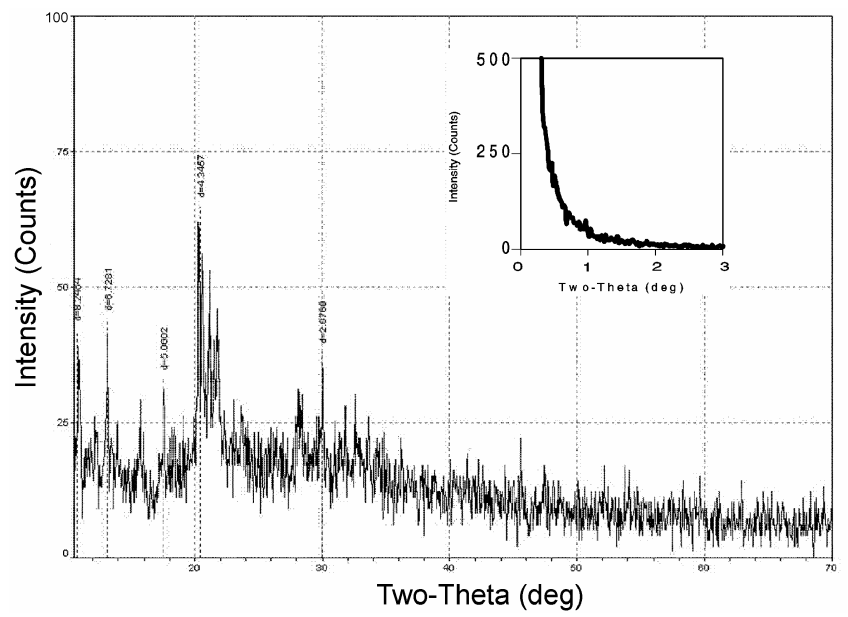

Figure 4. Wide angle X-ray diffraction with small angle Xray diffraction shown in inset. 
Table 1. Size and distribution of zinc nanoparticles.

\begin{tabular}{lccc}
\hline S1. no. & \% Abundance & Dia. $(\mathrm{nm})$ & Av. dia. $(\sigma)$ \\
\hline 1 & 65.9 & $9 \cdot 4$ & \\
2 & 21.0 & $20 \cdot 7$ & $14 \cdot 71 \backslash 8 \cdot 1$ \\
3 & $13 \cdot 0$ & $32 \cdot 1$ & \\
\hline
\end{tabular}

$\sigma$ represents the standard deviation in $\mathrm{nm}$.

at $370 \mathrm{~nm}$, which appears due to the formation of zinc oxide nanoparticles in colloidal solution having a gap of $3.4 \mathrm{eV}$ between valence and conduction bands. When high energy laser pulse falls on the metal surface it vapourizes zinc atoms, which reacts with dissolved oxygen in water to form $\mathrm{ZnO}$ molecule. Large numbers of $\mathrm{Zn} / \mathrm{ZnO}$ atoms $\backslash$ molecules arrange themselves in a particular geometry to synthesize $\mathrm{Zn} / \mathrm{ZnO}$ nanoparticles, which are encapsulated by a layer of surfactant to prevent agglomeration and terminates particle growth. This provides novel techniques for the production of metallic oxide nanoparticles in solution by increasing the amount of dissolved oxygen in it. Passing pure nitrogen or argon in solution to eliminate the dissolved oxygen from it, can be used to synthesize pure metallic nanoparticles.

The particles thus produced in the solution remain in colloidal form for several months. From table 1, it can be seen that about $66 \%$ of zinc nanoparticles are $9.4 \mathrm{~nm}$ in size with an average diameter of $14.71 \mathrm{~nm}$ and a standard deviation $(\sigma)$ of $8.1 \mathrm{~nm}$. The surface plasmon absorption spectrum of zinc nanoparticles in the solution has a single peak centred at $232.4 \mathrm{~nm}$, which indicates that zinc nanoparticles are spherical in shape. Earlier, it was reported that ellipsoidal (Petit et al 1993) and cylindrical (Link and El-Sayed 1999) nanoparticles exhibited two peaks in the absorption spectrum.

\section{Conclusions}

In the present study a novel technique, free from environmental pollution, is obtained to produce metallic and metallic oxide nanoparticles in the solution, which can remain stable for several months. The laser ablation of zinc plate in the aqueous solution of SDS is employed to prepare size and shape controlled nanoparticles. Plasmon absorption of zinc nanoparticles shows that they are potential candidates for wide band gap $(5.33 \mathrm{eV})$ semiconducting and ultra-violet lasing materials. They can also be used as good insulating material by further reduction in size using ablation and fragmentation. These nanoparticles are characterized using optical absorption, TEM, small angle Xray diffraction and wide angle $\mathrm{X}$-ray diffraction. Particles synthesized by this method have smaller size and distribu- tion as compared to the conventional chemical reduction method. The shape of the particles does not change after continuous laser irradiation on the solution as reported in the case of other metals and alloys like silver (Abid et al 2001), gold (Sibbald et al 1996), platinum (Lee et al 2001), silver-gold (Mafune et al 2000) and cobalt-platinum (Rakshit and Budhani 2006) nanoparticles. Termination in the growth of nanoparticles is being achieved by SDS added in water initially.

\section{Acknowledgements}

We are thankful to Prof. O N Srivastava, Department of Physics, Banaras Hindu University, Varanasi, for TEM spectrum, Prof. R C Budhani, Indian Institute of Technology, Kanpur, for helpful discussion and Nanophosphor Application Centre, Physics Department, Allahabad University, Allahabad, for X-ray diffraction.

\section{References}

Abid J P, Girault H H and Brevet P F 2001 Chem. Commun. 829

Darugar Q, Qian W and El-Sayed M A 2006 J. Phys. Chem. B110 143

Eastman J A, Choi S U S, Li S, Yu W and Thompson L J 2001 Appl. Phys. Lett. 78718

Fujiwara H, Yanagida S and Kamat P V 1999 J. Phys. Chem. B103 2589

Geohegan D B, Puretzky A A, Duscher G and Pennycook S J 1998 Appl. Phys. Lett. 722987

Henglein A, Gutierrez M, Janata E and Ershov B G 1992 J. Phys. Chem. 964598

Hodak J H, Henglein A, Giersig M and Hartland G V $2000 \mathrm{~J}$. Phys. Chem. B104 11708

Huang M H et al 2001 Science 2921897

Kuang W, Fan Y and Chen Y 2000 Langmuir 165205

Lee I, Han S and Kim K 2001 Chem. Commun. 178

Li C M, Lei H, Tang Y J, Luo J S, Liu W and Chen Z M 2004 Nanotechnol. 151866

Link S and El-Sayed M A 1999 J. Phys. Chem. B103 8410

Ma X, Yan Z, Yuan B and Li B 2005 Nanotechnol. 16832

Macdonald I D G and Smith W E 1996 Langmuir 12706

Mafune F, Kohno J, Takeda Y and Kondow T 2000 J. Phys. Chem. B104 9111

Maye M M, Lou Y and Zhong C 2000 Langmuir 167520

Mulvaney P, Giersig M and Henglein A 1993 J. Phys. Chem. 97 7061

Petit C, Lixon P and Pileni M P 1993 J. Phys. Chem. 9712974

Rakshit R K and Budhani R C 2006 J. Phys. D; Appl. Phys. 39 1743

Sibbald M S, Chumanov G and Cotton T M 1996 J. Phys. Chem. 1004672

Singh S C and Gopal R 2006 Proc. of sixth DAE-BRNS NLS-06, RRCAT, Indore pp 186-187 